\title{
Research on Automatic Detection System Based on Full face Pavement Crack Digital Image
}

\author{
Li Bian ${ }^{1, *}$, Baolin $\mathrm{Zhu}^{2}$, Jing $\mathrm{Chen}^{3}$ and Xiaohui $\mathrm{Ju}^{4}$ \\ ${ }^{1,4}$ China Academy of Transportation Sciences 616, Huixinli 240\#, Chaoyang District, Beijing, China \\ ${ }^{2}$ China Academy of Transportation Sciences 617, Huixinli 240\#, Chaoyang District, Beijing, China \\ ${ }^{3}$ China Academy of Transportation Sciences 620, Huixinli 240\#, Chaoyang District, Beijing, China
}

Received 15 May 2013; Accepted 25 July 2013

\begin{abstract}
Automatic detection system has been widely used in pavement detection. This paper describes the principle of shooting width adjustment and method of adjusting the width of the image acquisition. This system increased width of pavement video acquisition system from maximum width of 5 meters at present to full face pavement 12 meters.It is proved through testing sections that it achieved good image effect and met the design requirements.
\end{abstract}

Keywords: Full face detection system, Automatic detection system, Full face pavement, Adjusting the width of the image acquisition

\section{Introduction}

As a common damage in the freeway, pavement crack is the key point of highway maintenance. Since the traditional artificial detection method never fits for the demand of highway development[1]. Because the advantages of high accuracy, little influence on traffic and less human factors, automatic detection system has been widely used in pavement detection [2].Some typical systems include Japan's Komatsu system,Swedish PAVUE system,WiseCrax system developed by RoadWare company of Canada,DHDV realtime pavement crack detction system developed by Arkansas University of the Americas, The UK'S HARRIS systems, pavement detction system developed by ARRB, automatic pavement detection system RoadCrack developed by Australia NSW highway traffic bureau and CRISO company. Some typical systems in china include detction system developed by Nanjing University of Science and Technology, SINC-RTM pavement detction system developed by Wuhan University, 'ZOYON-RTM0' intelligent road testing and measurement system, highway pavement laser detection system developed by Chang.an University. CiCS developed by research institue of highway ministry of transport,etc. Some overseas system can automatic detection and analysis on the crack of $1 \mathrm{~mm}$ or above, the detection rate can reach $85 \%$ to $90 \%$,with high precision and high speed,the widest of detection pavement up to $5 \mathrm{~m}[1,2,3]$. Some domestic detection precision can reach $1 \mathrm{~mm}$, he widest of detection pavement up to $3.6 \mathrm{~m}$. Compared with foreign detection system,we are still have shortcomings in width of detection.

\section{Shooting width adjustment principle $[4,5]$}

\footnotetext{
* E-mail address: lisa2019@163.com

ISSN: 1791-2377 @ 2013 Kavala Institute of Technology. All rights reserved.
}

Full face detection system realized one lane $(4 \mathrm{~m})$ and the full face pavement (12 meters) for different precision image detail capture, playback, search. It can be associated with the image information, the time of the GPS real-time transmission and location information. The shooting angle in detecting means depression angle of imaging lens on the road,the camera angle of field of view. This system is mainly for one lane and the full face lane image information acquisition and processing. When collecting images, you need to projected objective world scene onto the image plane of the camera. This projector can be used to describe the camera model. It reflects the objects transformation between objective coordinate system, the camera coordinate system and photo coordinate system. Camera coordinate system shown in Figure 1.

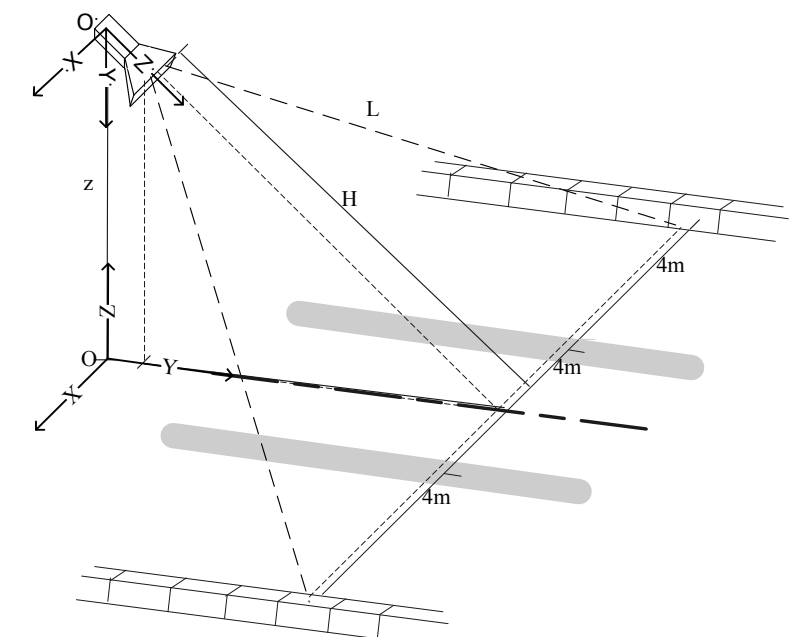

Fig.1. Camera coordinate system 
In Figure 1, OXYZ is objective coordinate system, $\mathrm{O}^{\prime} \mathrm{X}^{\prime} \mathrm{Y}^{\prime} \mathrm{Z}^{\prime}$ is camera coordinate system, also known as photo coordinate system. $Z^{\prime}$ axis is the optical axis of the camera.The position deviation between the center of image plane and objective coordinate system is D.It projected to the coordinate for the Dx, Dy, Dz. Assuming the camera scan the road with $\gamma$ angle (horizontal rotation) and $\alpha$ angle(vertical rotation).$\gamma$ is the angle between the $X$ axis and $X^{\prime}$ ' axis, $\alpha$ is the angle between the $Z$ axis and $Z$ ' axis. $\mathrm{W}(\mathrm{X}, \mathrm{Y}, \mathrm{Z})$ is the object to be photographed in objective coordinate system. $\mathrm{C}(\mathrm{x}, \mathrm{y}, \mathrm{z})$ is the object to be photographed in camera coordinate system. $f$ is the focal length of the camera. The camera coordinate system rotation transformation, so that it coincides with the objective coordinate axes. Based on the perspective principles, it gets relationship between $\mathrm{W}$ and $\mathrm{C}$.

$\mathrm{C}=\mathrm{PTRW}(\mathrm{P}$ is perspective relationship matrix, $\mathrm{T}$ is the translational relationship matrix, $\mathrm{R}$ is the rotation matrix).

$P=\left(\begin{array}{cccc}1 & 0 & 0 & 0 \\ 0 & 1 & 0 & 0 \\ 0 & 0 & 1 & 0 \\ 0 & 0 & -1 / f & 0\end{array}\right)$

$T=\left(\begin{array}{cccc}1 & 0 & 0 & -D x \\ 0 & 1 & 0 & -D y \\ 0 & 0 & 1 & -D z \\ 0 & 0 & 0 & 1\end{array}\right)$

$R=\left(\begin{array}{cccc}\cos \gamma & \sin \gamma & 0 & 0 \\ -\sin \gamma \cos \alpha & \cos \alpha \cos \gamma & \sin \alpha & 0 \\ -\sin \gamma \sin \alpha & -\sin \alpha \cos \gamma & \cos \alpha & 0 \\ 0 & 0 & 0 & 1\end{array}\right)$

As the camera three-dimensional image of the objective is photographed in two-dimensional image plane,so $\mathrm{z}$ value does not exist. It can be obtained in equation 1 that the objective C Cartesian coordinate system (x, y) expressed as equation 2 .

$$
\left\{\begin{array}{l}
x=f \frac{(X-D x) \cos \gamma+(Y-D y) \sin \gamma}{-(X-D x) \sin \alpha \sin \gamma+(Y-D y) \sin \alpha \cos \gamma-(Z-D z) \cos \alpha+f} \\
y=f \frac{-(X-D x) \sin \gamma \cos \alpha+(Y-D y) \cos \alpha \cos \gamma+(Z-D z) \sin \alpha}{-(X-D x) \sin \alpha \sin \gamma+(Y-D y) \sin \alpha \cos \gamma-(Z-D z) \cos \alpha+f}
\end{array}\right.
$$

As shown in equation 2, if the objective coordinate system and camera coordinate systems do not coincide, it have a non-linear relationship between $\mathrm{X} / \mathrm{Y}$ and $\mathrm{x} / \mathrm{y}$. So the image projection transformation relation is complex, image deformation distortion is serious,post-processing and correction of image become heavy workload. When $\mathrm{Dx}=\mathrm{Dy}=\mathrm{Dz}=0, \gamma=\alpha=0^{\circ}$, objective coordinate system and the camera coordinate system coincides, equation 2 simplifies to equation 3 .

$$
\left\{\begin{array}{l}
x=\frac{f X}{f-Z} \\
y=\frac{f Y}{f-Z}
\end{array}\right.
$$

$\mathrm{Z}$ represents the object distance. If $\mathrm{Z}$ is the set value, it is a fixed ratio between $\mathrm{x}$ and $\mathrm{X}, \mathrm{y}$ and Y.It Is equivalent to the objective world scaled down image. When installing a CCD camera, if the axis perpendicular to the road surface,the image without distortion,this is the best quality. When the camera lens height $\mathrm{z}$ is fixed,the camera angles very useful to increase the shooting range.Because each pixel corresponds to a resolution expanded,it decreased sharpness of the image obtained.Shooting angle decreases obtain a clear image in detail,but reduces camera range. When adjusting the camera angle,vertical rotating camera can be obtained under the road width requirements, it can reducing image distortion. In addition, the line scan camera image acquisition process can ignore the impact of the $\mathrm{Y}$-axis direction of deformation (camera exposure and traffic speed matching cases). Equation 4 is a vertical rotation angle adjustment formula image map (without the edges of the lens distortion).

$$
\left\{\begin{array}{l}
x=f \frac{X}{Y \sin \alpha-Z \cos \alpha+f} \\
y=f \frac{Y \cos \alpha+Z \sin \alpha}{Y \sin \alpha-Z \cos \alpha+f}
\end{array}\right.
$$

\section{Method of adjusting the width of the image acquisition}

Full face detection system include CCD camera,host computer data processing components, etc. Hardware of detection system shown in Figure 2.

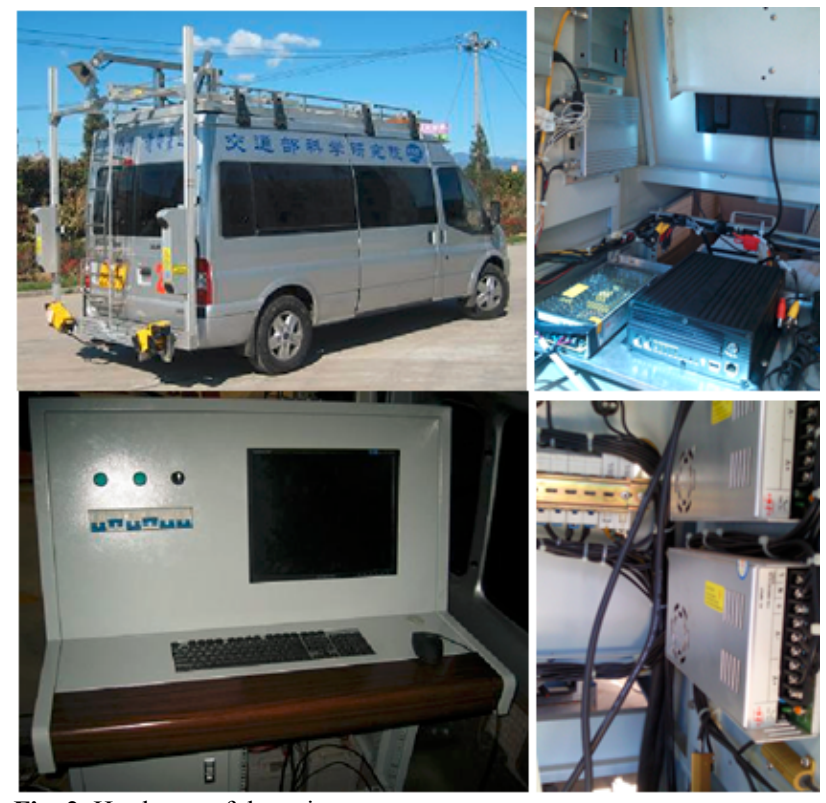

Fig. 2. Hardware of detection system

According to the principle of adjusting the angle, the camera mounting plate produced a stopper bolt .It shown in Figure 3. It can precisely define the scope camera, but also reduce the vibration of the camera during driving. Spacing hole rotate on arc distribution of nine pairs. The 1 st hole is to shoot the full face pavement (about 12 meters), an angle of approximately $75^{\circ}, \mathrm{Z}=2.6 \mathrm{~m}, \mathrm{X}=9.5 \mathrm{~m}$. The 4 th hole shoot one lane (about 4 meters), an angle of approximately $40^{\circ}$, $\mathrm{Z}=2.6 \mathrm{~m}, \mathrm{X}=2.1 \mathrm{~m}$. 


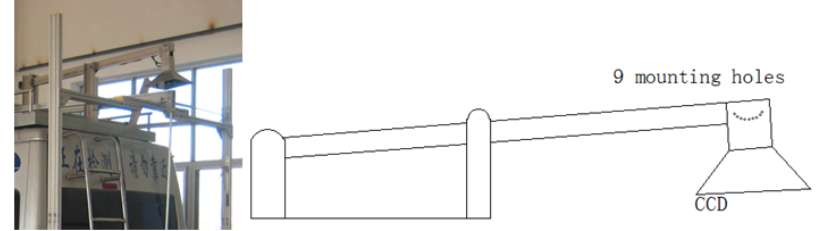

Fig.3 Camera installation method

Figure 4 is a static image, it capture width of $12 \mathrm{~m}$ in the left, middle and right position of the calibration map. Little change in the calibration board resolution, angle adjustment reasonable. The edge of light is dimmer than light in middle,because the lens edge absorb less light. System can gain light compensation through hardware or software enhancements. Full face pavement static calibration image shown in Figure 4.
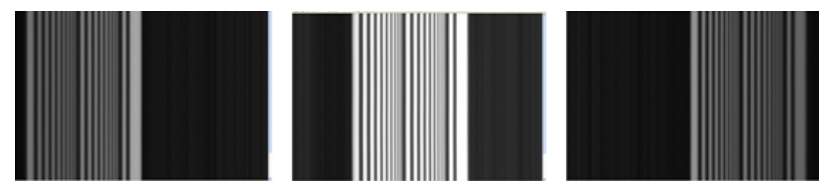

Fig.4. Full face pavement static calibration image

Under external trigger mode, because the revolution of the wheel sensor output pulse little, it is not enough to make the camera to record the complete pavement image. It requires the Hall sensor output signal frequency doubling, it is known as the frequency multiplication. Since the multiplier is a frequency multiplication in binary, it also needs to be converted decimal number into binary number. Multiplier number is calculated as shown in Equation 5.

Multiplier $\mathrm{M}=\frac{2.23 \times \text { Camera resolution } \mathrm{N}}{\text { image widthW } \times 13.4}-1$

For example, if a 2048 pixel line camera scan $12 \mathrm{~m}$ width pavement for image acquisition, the multiplier should be in Equation 6.

Multiplier $\mathrm{M}=\frac{2.23 \times 2048}{12 \times 13.4}-1=27$ (times)

So it should set the multiplier number is 27 ,with the binary representation is 00011011 .

\section{Data acquisition and the resulting output}

Utilization full face detection system opens a video image. It identifies transverse crack, longitudinal joint,alligator cracking, net-shaped cracking .etc.Interface of crack length and area measurements as shown in Figure 5.

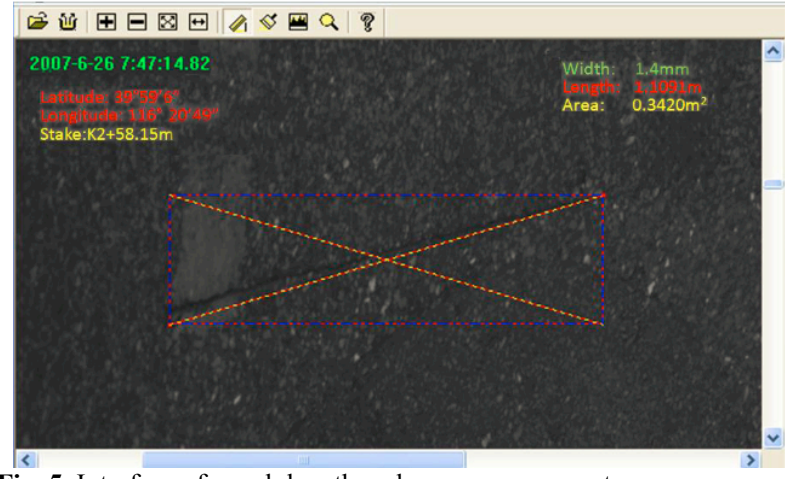

Fig. 5. Interface of crack length and area measurements

Statistical results can be simultaneously output format with EXCEL format and txt file format. Output results as shown in Figure 6.Various forms of pavement distress are classified and saved in different names of txt document.It will facilitate inquiries and classify. Txt document will be classified into another evaluation system. It analyzes the data according to different road sections, different directions road and overall road. It calculates riding quality index(RQI) and pavement surface condition index(PCI). It can print out overall report and subreport of the data and results of the evaluation.

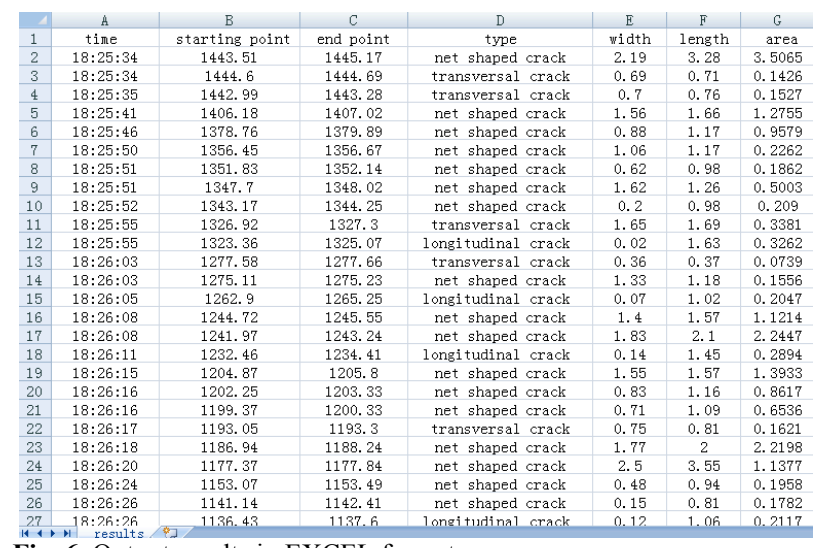

Fig. 6. Output results in EXCEL format

\section{Conclusions}

The developing of image sampling system is the key of this system.Through camera angle adjustment and the Hall sensor output signal frequency doubling, It increased width of pavement video acquisition system from maximum width of 5 meters at present to full face pavement 12 meters. It achieved good results in increased detection width. But the detection accuracy is affected,it aslo requires further study. This system can save road surface crack image to the hardware of computer completely, which can used in the later image processing. It is proved through testing sections that it achieved good image effect and met the design requirements. Research result facilitates the development of pavement automatic detection and ITS.

\section{References}

1. Yi Lin, The Design and Realization of Image Processing in Vehicle-mounted Pavement Crack Detection System, Wuhan: Huazhong University of Science \& Technolog, 2011.
2. Xiaoyu Dou, Research on The Asphalt Pavement Crack Automatic Detection Technology Based on Multi-scale Analysis, Xi.an: Chang.an University, 2010. 
3. Changrong Xie, The Design and Research of Road Surface Cracks Detection System, Xi.an: Chang.an University, 2008.

4. Yanping Jing,Daoxin Wei,Xiaohui Ju,Baolin Zhu,Li Bian,Wanli Yang.Treatment of High-Performance Asphalt Pavement Cracks Conservation Technology Research[R]. Beijing: China Academy of Transportation Sciences, 2008.

5. Bin Hu,Haiwen Yuan,Yunsong Wu,Hao Yang,Baolin zhu. Design and Realization of Vehicle Borne Test System for the Highway Cracks. Measurement \& Control Technology.2008,27(5):58-61.

6. Yunsong $\mathrm{Wu}$,Haiwen Yuan, Yanping Jing,Baolin Zhu,Hao Yang. Automatic Pavement Crack Identification System Based on Digital Image Processing, Control and application of the Thirteenth Annual Symposium, 2008, pp.6-17.

7. Jieqiong Xu. Research on Vehicle Detection and Tracking Method Based on Video Image Processing,Qingdao: Ocean University of China,2012.

8. Liangjun He. Study on the Detection System of Asphalt Pavement Crack Based on Image Processing, Xi.an: Chang.an University, 2008.
9. Yanli Zhu. Automatic Pavement Crack Identification System Research Based on Digital Image Processing, Xi.an: Chang.an University, 2008

10. Xiaofei Li. Research on Pavement Crack Detection Algorithom Base on Digital Image Processing, Nanjing:Nanjing University of Post and Telecommunications, 2012.

11. Hanxing Qiu. Study of Pavement Crack Detection and Idetification Base on Image Segmentation,Chongqing:ChongqingJiaotong University, 2012

12. Changxia Ma. Researeh on the Key Technologies of Pavement Crack Detection Based On Image Analysis,Nanjing: Nanjing University of Science\&Technology, 2012.

13. Juan Zhang,Ai-min Sha,Huaigang Gao,Chaoyun Sun. Automatic pavement crack recognition and evaluation system based on digital image processing. Journal of Changpan University (Natural Science Edition).2004,24(2):18-22. 